\title{
III. On the relation between the velocity and length of a wave, in the undulatory theory of light
}

\author{
John Tovey Esq.
}

To cite this article: John Tovey Esq. (1836) III. On the relation between the velocity and length of a wave, in the undulatory theory of light, Philosophical Magazine Series 3, 8:43-44, 7-12, DOI: $10.1080 / 14786443608648788$

To link to this article: http://dx.doi.org/10.1080/14786443608648788

曲 Published online: 21 Aug 2009.

Submit your article to this journal $[\pi$

Џll Article views: 2

Q View related articles ¿ 
the dental edge truncated and square; the superior maxillary is irregularly triangular, much elongated, and contracts insensibly towards its dental extremity, which is bifid.

In the Chimara Agassizii of Dr. Buckland the inferior maxillary is the most regular in form of the four species; it is nearly square, and has the dental edge slightly open ; the surface of the symphysis is flatter than in the other species.

The Chimara Mantellii has the inferior jaw straighter and thinner: its exterior surface is perfectly smooth and flat; its snout is much elongated and pointed, and the cavity of the dental edge wider.

Since Dr. Buckland's discovery of the above four species, I have found a fifth in the collection of Mr. Greenough, which differs considerably from them all, in the extreme shortness of the lower jaw, the length of which is less than its height. The symphysis of the lower jaw is flat; the dental margin truncated and grooved in its hinder part. The external surface is smooth; the middle of the inner surface concave; the intermaxillary is flatter than in the Chimcera Egertonii, and terminates in a straight point. The superior maxillary is shorter than that of the Chimera Egertonii.

I propose to give to this species of so remarkable a genus the name of Chimcera Greenovii. The locality of this fossil is unknown.

Oxford, Oct. 27, 1835.

III. On the Relation betroeen the Velocity and Length of a Wave, in the Undulatory Theory of Light. By JoHN Tover, Esq.

To the Editors of the Philosophical Magazine and Journal. Gentremen,

IN the last volume but one of your Magazine, the Rev. Professor Powell presented us with an abstract of the essential principles of M. Cauchy's View of the Undulatory Theory of Light; by which, as Mr. Powell says, it appears " that a relation between the velocity and length of a wave is established on M. Cauchy's principles, provided the molecules are so disposed that the intervals between them always bear a sensible ratio to the length of an undulation." vol. vi. p. 266.

Since I first read this, I have arrived at the same result as M. Cauchy by a less complicated method, which I proceed to lay before you. I do this with diffidence, having read scarcely anything on the subject besides the abstract above mentioned and Professor Airy's tract. Should you deem 
my method worthy of a place in your Journal, I shall probably send you a continuation of the subject.

Let $m, m^{\prime}, m^{\prime \prime}, \& c$. be the masses of the particles of æther; let the rectangular coordinates of $m$ be $x, y, z$; those of $m^{\prime}$, $x+\Delta x, y+\Delta y, z+\Delta z$; of $m^{\prime \prime}, x+\Delta x^{\prime}, y+\Delta y^{\prime}, z+\Delta z^{\prime}, \&$ c. Let $r=\sqrt{ }\left(\Delta x^{3}+\Delta y^{2}+z^{2}\right), r^{\prime}=\sqrt{ }\left(\Delta x^{\prime 2}+\Delta y^{\prime 2}+\Delta z^{\prime 2}\right)$, \&c. Suppose the masses to be all equal, and the force of one particle on another to be a function of their distance multiplied by $m$; and suppose each particle to be influenced only by the attractions or repulsions of the other particles; then as the cosines of the angles which $r$ makes with the positive directions of $x, y, z$ are $\frac{\Delta x}{r}, \frac{\Delta y}{r}, \frac{\Delta z}{r}$, we have (by the principles of statics), when the system is in equilibrium,

$$
\begin{gathered}
m \Sigma \cdot \frac{f(r)}{r} \Delta x=0, m \Sigma \cdot \frac{f(r)}{r} \Delta y=0, \\
m \Sigma \cdot \frac{f(r)}{r} \Delta z=0 .
\end{gathered}
$$

The sums $\Sigma$ extending to all the particles within the sphere of the attractive or repulsive influence of the particle $m$, which may be any particle of the system.

Now, suppose the system to be disturbed, and that at the end of the time $t$, the displacements of $m$, in the directions of $x, y, z$, be $\xi, \eta, \zeta$; and those of $m^{\prime}, \xi+\Delta \xi, \eta+\Delta \eta, \zeta+\Delta \zeta$; and suppose $\Delta \xi, \Delta \eta, \Delta \zeta$ to be so small that we may neglect their squares and rectangles; then the distance of these particles being $r+\Delta r=\sqrt{ }\left[(\Delta x+\Delta \xi)^{2}+(\Delta y+\Delta \eta)^{2}+(\Delta z+\Delta \zeta)^{2}\right]$, we have

$$
\Delta r=\Delta x \Delta \zeta+\Delta y \Delta \eta+\Delta z \Delta \zeta .
$$

The cosines of the directions which $r+\Delta r$ makes with those of $x, y, z$ will be $\frac{\Delta x+\Delta \xi}{r+\Delta r}, \frac{\Delta y+\Delta \eta}{r+\Delta r}, \frac{\Delta z+\Delta \zeta}{r+\Delta r}$; and if we write $\mathrm{X}, \mathrm{Y}, \mathrm{Z}$ for the sums of the components of the forces acting on $m$, in the directions of $x, y, z$, we have

$$
\begin{aligned}
& \mathrm{X}=m \Sigma \cdot \frac{f(r+\Delta r)}{r+\Delta r}(\Delta x+\Delta \xi), \\
& \mathrm{Y}=m \Sigma \cdot \frac{f(r+\Delta r)}{r+\Delta r}(\Delta y+\Delta \eta), \\
& \mathrm{Z}=m \mathrm{Z} \cdot \frac{f(r+\Delta r)}{r+\Delta r}(\Delta z+\Delta \xi) .
\end{aligned}
$$


Length of a Wave, in the Undulatory Theory of Light. 9

Now, $f(r+\Delta r)=f(r)+\frac{d f(r)}{d r} \Delta r, \frac{1}{r+\Delta r}=\frac{1}{r}-\frac{\Delta r}{r^{a}}$; consequently,

$$
\begin{aligned}
& \frac{f(r+\Delta r)}{r+\Delta r}(\Delta x+\Delta \xi)=\left\{\frac{f(r)}{r}+\left(\frac{d f(r)}{r d r}-\frac{f(r)}{r^{2}}\right) \Delta r\right\} \\
& \cdot(\Delta x+\Delta \xi) \\
& =\frac{f(r)}{r} \Delta x+\frac{f(r)}{r} \Delta \xi+\left(\frac{d f(r)}{r^{2} d r}-\frac{f(r)}{r^{3}}\right) \\
& \text {. }(\Delta x \Delta \xi+\Delta y \Delta \eta+\Delta z \Delta \xi) \Delta x \text {, }
\end{aligned}
$$

by substituting for $\Delta r$ its value previously found.

If in this expression we write $\phi(r)$ for $\frac{f(r)}{r}, \psi(r)$ for $\left(\frac{d f(r)}{r^{2} d r}-\frac{f(r)}{r^{3}}\right)$, and substitute it in the first of the equations (2.), we shall have by virtue of the first of the equations (1.), .

$$
\mathrm{X}=m \Sigma .\{\phi(r) \Delta \xi+\psi(r) \cdot(\Delta x \Delta \xi+\Delta y \Delta \eta+\Delta z \Delta \xi) x\} \cdot
$$

The second and third of the equations (2.) are similar to the first, consequently if we transform them in the same manner, and (by the principles of dynamics) put $\frac{d^{2} \xi}{d t^{2}}, \frac{d^{3} \eta}{d t^{2}}$, $\frac{d^{3} \zeta}{d t^{2}}$ for $\mathrm{X}, \mathrm{Y}, \mathrm{Z}$, we shall have

$$
\begin{aligned}
& \frac{d^{2} \xi}{d t^{2}}=m \Sigma \cdot\{\phi(r) \Delta \xi+\psi(r) \cdot(\Delta x \Delta \xi+\Delta y \Delta \eta+\Delta z \Delta \zeta) \Delta x\}, \\
& \frac{d^{2} \eta}{d t^{2}}=m \Sigma \cdot\{\phi(r) \Delta \eta+\psi(r) \cdot(\Delta x \Delta \xi+\Delta y \Delta \eta+\Delta z \Delta \zeta) \Delta y\}, \\
& \left.\frac{d^{2} \zeta}{d t^{2}}=m \Sigma \cdot\{\phi(r) \Delta \zeta+\psi(r) \cdot(\Delta x \Delta \xi+\Delta y \Delta \eta)+\Delta z \Delta \zeta) \Delta z\right\} .
\end{aligned}
$$

From these general equations, a number of integrals, adapted to particular cases, may be found. Let us suppose the vibrations of the particles to be performed in straight lines, all in one direction. This is a case of polarized light. Let $x$ be taken in the direction of the vibrations; then $\eta$ and $\zeta$ will be zero, and the first of the equations (3.) will give

$$
\frac{d^{\Sigma} \xi}{d t^{2}}=m \Sigma \cdot\left\{\phi(r)+\psi(r) \Delta x^{2}\right\} \Delta \xi .
$$

Third Series. Vol. 8. No. 43. Jan. 1836. 


\section{$10 \mathrm{Mr}$. Tovey on the Relation between the Velocity and} put

Now, let $\xi$ be a function of $z$ and $t$, then for $\Delta \xi$ we may

$$
\frac{d \xi}{d z} \Delta z+\frac{d^{2} \xi}{d z^{2}} \cdot \frac{\Delta z^{8}}{2}+\frac{d^{3} \xi}{d z^{3}} \cdot \frac{\Delta z^{3}}{2.3}+\frac{d^{4} \xi}{d z^{4}} \cdot \frac{d z^{4}}{2.3 .4} .
$$

If we substitute this value of $\Delta \xi$ in equation (4.), and suppose the particles to be so arranged in their state of equilibrium that for every particle on one side of $m$, within the sphere of its influence, there is another at an equal distance on the opposite side, we may divide the sum $\Sigma$ into two parts, one comprehending the positive values of $\Delta z$, and the other the negative; and for every term in one part we shall have a term equal to it, and involving the same power of $\Delta z$, in the other. But in the one part all the terms involving odd powers of $\Delta z$ will be positive, and in the other all negative; consequently all these terms will vanish: the other terms being all positive, the sum of one part will be equal to that of the other. Consequently equation (4.) will become

$$
\frac{d^{2} \xi}{d t^{2}}=m \Sigma \cdot\left\{\Phi(r)+\psi(r) \Delta x^{2}\right\} \Delta z^{2}\left(\frac{d^{2} \xi}{d z^{2}}+\frac{d^{4} \xi}{d z^{4}} \cdot \frac{\Delta z^{2}}{3.4}\right):
$$

the sum, in this equation, extending only to the positive values of $\Delta z$.

For $m \Sigma .\left\{\phi(r)+\psi(r) \Delta x^{2}\right\} \Delta z^{2}$, write $s^{2}$, and for $m \Sigma$. $\left\{\Phi(r)+\psi(r) \Delta x^{2}\right\} \Delta z^{4}$ write $s^{\prime 2}$, then the last equation will be

$$
\frac{d^{2} \xi}{d t^{2}}=s^{2} \cdot \frac{d^{2} \xi}{d z^{2}}+s^{\prime 2} \cdot \frac{d^{4} \xi}{d z^{4}} \text {. }
$$

If we omit the last term, this equation becomes exactly of the same form as that for the transmission of sound, and gives then no relation between the length and velocity of the waves. But if we integrate the equation as it is, we shall obtain a relation of this sort; and this relation will afford a theory of the dispersion of light.

As $\xi$ is a function of $z$ and $t$, it may be expressed by a series of terms such as $p \sin n t+q \cos n t$, where $p$ and $q$ are functions of $z$, and $n$ a constant quantity*. Suppose then $\xi=p \sin n t+q \cos n t$; substitute this value of $\xi$ in equation (5.), and it will become

$$
\left.\begin{array}{r}
\left(n^{2} p+s^{2} \cdot \frac{d^{2} p}{d z^{2}}+\frac{s^{2}}{3.4} \cdot \frac{d^{4} p}{d z^{4}}\right) \sin n t \\
+\left(n^{2} q+s^{2} \cdot \frac{d^{2} q}{d z^{2}}+\frac{s^{2}}{34} \cdot \frac{d^{4} q}{d z^{4}}\right) \cos n t
\end{array}\right\}=0 .
$$

* Poisson, Traité de Mécanique, No. 514, edit. 2. 
This equation must be true for all values of $t$, therefore

$$
\begin{aligned}
& n^{2} p+s^{2} \cdot \frac{d^{2} p}{d z^{2}}+\frac{s^{\prime}}{3.4} \cdot \frac{d^{4} p}{d z^{4}}=0 \\
& n^{2} q+s^{2} \cdot \frac{d^{2} q}{d z^{2}}+\frac{s^{\prime 2}}{3.4} \cdot \frac{d^{4} q}{d z^{4}}=0 .
\end{aligned}
$$

Now, as $p$ is a function of $z$, it may be expressed by a series of such terms as $a \sin k z+b \cos k z$, where $a, b$, and $k$ are constant quantities. Substitute $a \sin k z+b \cos k z$ for $p$ in equation (6.), and it will become

$$
\begin{gathered}
n^{2}-s^{2} k^{2}+\frac{s^{2}}{3.4} k^{4}=0 ; \text { hence } \frac{n}{k} \\
=\sqrt{\left(s^{2}-\frac{s^{2}}{3.4} k^{2}\right)=s\left(1-\frac{k^{2}}{2.3 .4} \cdot \frac{s^{\prime 2}}{s^{2}}\right), \text { nearly. }}
\end{gathered}
$$

As the equations (6.) and (7.) are similar, and as we have put $a \sin k z+b \cos k z$ for $p$, we must put $a^{\prime} \sin k z+b^{\prime} \cos$ $k \approx$ :for $q ; a^{\prime}$ and $b^{\prime}$ being two more constant quantities. Hence $\xi$ may be expressed by a series of terms similar to

$(a \sin k z+b \cos k z) \sin n t+\left(a^{\prime} \sin k z+b^{\prime} \cos k z\right) \cos n t$.

With respect to any particular value of $z$, this term goes through all its values while $t$ increases by $\frac{2 \pi}{n}$; and with respect to any particular value of $t$, it goes through all its values while $z$ increases by $\frac{2 \pi}{k}$; consequently it represents a wave of light, moving in the direction of $z$, with the velocity $\frac{n}{k}$, the value of which has just been found equal to

$$
s\left(1-\frac{k^{2}}{2 \cdot 3 \cdot 4} \cdot \frac{s^{\prime 2}}{s^{2}}\right) \text {. }
$$

Professor Powell's expression for this quantity is

$\mathbf{H}\left\{\frac{\sin \left(\frac{\pi r n}{l}\right)}{\frac{\pi r n}{l}}\right\}$, which is equal to $\mathrm{H}\left(1-\frac{\pi^{2} r^{2} n^{2}}{2.3 l^{2}}\right)$, very nearly. As the Professor considers only one term instead of the sums $s^{2}$ and $s^{\prime 2}$, and as $r n$ and $l$ in his notation are the same as $\Delta z$ and $\frac{2 \pi}{k}$ in ours, the two expressions are virtually the same.

$$
\text { C. } 2
$$


If we examine the composition of the quantity $\frac{s^{\prime 2}}{s^{2}}$, we shall see that the relation between the length and velocity of a wave does not depend merely upon the ratio of the intervals of the particles to the length of an undulation, but also upon the radius of the sphere of their influence.

On the last principle, I think we can (as M. Fresnel seems to have conjectured*) account for the dispersion of light without supposing that the waves move with different velocities in the free æetherial medium; to which supposition there seems to be an insuperable objection $t$. But I reserve this for future consideration. In the mean time 1 hope Professor Powell will favour us, through the medium of your Journal, with what he has done towards the verification of M. Cauchy's formula, having told us that he is engaged on the subjectf.

Evesham, Aug. 17, 1835. I am, yours, \&c.

P.S. In my next communication I believe I shall be able to show that if the vibrations of the particles of æther be ench decomposed into three rectangular directions, two of which are perpendicular to the direction in which the light is propagated, and the other parallel to it, the vibrations in any one of these three directions may be calculated separately; and that a satisfactory reason may be assigned why the vibrations in the direction of propagation are insensible. (Airy's 'Tract, art. 101.)

September 3, 1835.

IV. Extracts from a Prize Essay on Iodine. By JaMes
INGIS, M.D. [Continued from p. 444, and concluded.]

V. COURTOIS shortly after his discovery of iodine I1. formed the black pulverulent iodide of nitrogen by the action of iodine upon hydrous ammonia. Gay-Lussac next described another compound of dry ammoniacal gas and iodine, which he called the ioduret of ammonia, but which might rather be called a hydriodate of an iodide of nitrogen. When this compound is thrown into water, decomposition takes place, hydriodic acid is found in the water, and the black iodide

* Airy's 'Tracts, p. 285, note.

+ Ibid.

[+ Notices of Professor Powell's verifications of M. Cauchy's modification of the undulatory theory, so far as they have yet been made public, will be found in Lond. and Edirb. Phis. Mag., vol. vi. p. 374; and last volune, p. 293.-Eorr.] 